\title{
Implante unitário com função imediata após extração: relato de caso
}

\author{
Single implant with immediate function after extraction: case report \\ Implante único con función immediata tras la extracción: relato de caso
}

\author{
Ricardo Seixas de Paiva Lima \\ ORCID: https://orcid.org/0000-0002-6322-6504 \\ Universidade Santo Amaro, Brasil \\ E-mail: ricardoseixasdepaiva@gmail.com \\ Gustavo Seixas de Paiva Lima \\ ORCID: https://orcid.org/0000-0002-1075-7643 \\ Odontocentro prof. Javan Paiva, Brasil \\ E-mail:gs.odonto@gmail.com \\ Caio Vinícios Gonçalves Roman Torres \\ ORCID: https://orcid.org/0000-0001-9864-6894 \\ Universidade Santo Amaro, Brasil \\ E-mail: Cvgrt@hotmail.com \\ Angélica Castro Pimentel \\ ORCID: https://orcid.org/0000-0002-6317-6439 \\ Universidade Santo Amaro, Brasil \\ E-mail: draangelicacp@uol.com.br \\ Wilson Roberto Sendyk \\ ORCID: https://orcid.org/0000-0002-3742-1330 \\ Universidade Santo Amaro, Brasil \\ E-mail: wilsen@ terra.com.br \\ Heloisa Fonseca Marão \\ ORCID: https://orcid.org/0000-0002-6128-8576 \\ Universidade Santo Amaro, Brasil \\ E-mail: hfmarao@prof.unisa.br
}

\begin{abstract}
Resumo
A reabilitação de um paciente com algum tipo de perda dentária deve ser realizada o mais rápida possível, causando assim menos problemas estéticos e funcionais ao paciente. O implante dentário com função imediata, é uma ótima alternativa para diminuir o tempo de espera da reabilitação em relação à a técnica convencional, sendo colocada a prótese logo após a instalação do implante, com resultados similares ao da técnica convencional. Além disto, existe maior preservação do osso periimplantar como também do contorno gengival. Uma das indicações para o uso da técnica é quanto ocorre fratura dental. Portanto, o objetivo do trabalho foi descrever a técnica realizada em um caso clínico, no qual o paciente foi reabilitado com implante em função imediata, após a fratura do primeiro pré-molar superior, sendo removido o elemento 24 e instalado 1 implante. A técnica utilizada foi a de ósteo expansão juntamente com enxerto para preenchimento do GAP. Com a obtenção de um torque de $45 \mathrm{~N}$.cm foi possível reestabelecer a função e estética imediata da região. Foi concluído que a função imediata com o uso de implantes dentários após exodontia é possível, sendo uma técnica favorável que proporcionou o sucesso na reabilitação, devolvendo a função e estética imediata ao paciente.
\end{abstract}

Palavras-chave: Implante dentário; Exodontia; Prótese dentária fixada por implante.

\begin{abstract}
The rehabilitation of a patient with some type of tooth loss must be carried out as quickly as possible, thus causing fewer aesthetic and functional problems for the patient. Dental implants with immediate function are an excellent alternative to reduce the waiting time for rehabilitation in relation to the conventional technique, with the prosthesis being placed soon after the installation of the implant, with results similar to those of the conventional technique. In addition, there is greater preservation of the peri-implant bone as well as the gingival contour. One of the indications for using the technique is when dental fracture occurs. Therefore, the objective of this study was to describe the technique performed in a clinical case, in which the patient was rehabilitated with an implant in immediate function, after the fracture of the upper first premolar, removing element 24 and installing 1 implant. The technique used was bone expansion together with a graft to fill the GAP. By obtaining a torque of $45 \mathrm{~N} . \mathrm{cm}$, it was possible to re-establish the immediate function and aesthetics of the region. It was concluded that immediate function with the use of dental implants after extraction is possible, being a favorable technique that provided success in rehabilitation, returning immediate function and esthetics to the patient.
\end{abstract}

Keywords: Dental implant; Extraction; Implant-attached dental prosthesis. 


\begin{abstract}
Resumen
La rehabilitación de un paciente con algún tipo de pérdida dentaria debe realizarse con la mayor rapidez posible, provocando así menos problemas estéticos y funcionales para el paciente. Los implantes dentales con función inmediata son una excelente alternativa para reducir el tiempo de espera para la rehabilitación en relación a la técnica convencional, colocándose la prótesis poco después de la instalación del implante, con resultados similares a los de la técnica convencional. Además, existe una mayor conservación del hueso periimplantario así como del contorno gingival. Una de las indicaciones para utilizar la técnica es cuando se produce una fractura dentaria. Por tanto, el objetivo de este estudio fue describir la técnica realizada en un caso clínico, en el que el paciente fue rehabilitado con un implante en función inmediata, tras la fractura del primer premolar superior, retirando el elemento 24 e instalando 1 implante. La técnica utilizada fue la expansión ósea junto con un injerto para rellenar el GAP. Al obtener un torque de $45 \mathrm{~N} . \mathrm{cm}$, fue posible restablecer la función inmediata y la estética de la región. Se concluyó que es posible la función inmediata con el uso de implantes dentales después de la extracción, siendo una técnica favorable que brindó éxito en la rehabilitación, devolviendo la función y estética inmediata al paciente.
\end{abstract}

Palabras clave: Implante dental; Extracción; Prótesis dental unida a implante.

\title{
1. Introdução
}

Os implantes dentários são utilizados desde a década de 60, através de uma técnica descrita por Branemark, obtendo excelentes resultados ao longo dos anos (Branemark et al., 1969). A técnica é preconizada com duas intervenções cirúrgicas (2 estágios cirúrgicos), a primeira para a instalação do implante dentário e a segunda para a reabertura, dando acesso ao implante, podendo desta maneira instalar a prótese (Adell, et al., 1981; Bahat, 2000). Porém, observou-se a necessidade de resultados mais rápidos, o que foi obtido com a carga imediata sobre o implante, diminuindo para apenas 1 tempo cirúrgico. Desta forma, diversos trabalhos foram publicados, demonstrando que é possível colocar a prótese sobre implante no mesmo momento da cirurgia de instalação do implante, sendo uma técnica tão aceitável e previsível quando comparada a técnica convencional de 2 estágios (Bassir, et al., 2019; Ericsson, et al., 2000; Piattelli, et al., 1993; Popper, et al., 2003).

O sucesso das próteses dentárias suportadas por implantes depende de um contexto geral, como a seleção do paciente em relação à saúde geral e condição sistêmica, a estabilidade primária e secundária do implante e o manejo adequado dos tecidos moles. Além disso, para se obter o sucesso com carga imediata em elementos unitários, alguns critérios devem ser respeitados, como o controle da quantidade de carga, a densidade óssea, a superfície do implante, bem como a própria técnica cirúrgica (Piattelli, et al., 1993; Andersen, et al., 2002; Haas, et al., 1995). Um fator essencial, para se obter sucesso na carga imediata, é alcançar um torque de no mínimo 35N.cm (Juodzbalys \& Wang, 2010).

São vantagens da função imediata sobre implantes: preservação do osso peri-implantar, em relação a técnica tradicional (Bassir, et al., 2019), como também preservação do contorno gengival ( Brunski, 1992; Becker, 2006; Leary, 2003). Quanto mais rápido for feita a instalação de implantes após a extração dentária, mudanças favoráveis nos tecidos ósseos e gengivais podem ocorrer, desta forma melhorando a preservação da anatomia óssea (Schropp, et al., 2003).

Sendo assim, o objetivo do trabalho foi descrever um caso clínico, no qual foi instalado 1 implante, após exodontia, na região de primeiro pré-molar superior, para reestabelecer a função imediata da região, relatando a técnica utilizada.

\section{Metodologia}

Paciente SMM com 67 anos, sexo feminino, leocoderma, compareceu em consultório particular (Odontocentro Prof. Javan Paiva, Recife, PE, Brasil), com o elemento dentário 24 fraturado. O paciente relatou que, o dente havia quebrado enquanto a mesma se alimentava. Ao exame clínico, foi constatada a fratura dentária (Figura 1). 
Figura 1: Foto inicial demonstrando a fratura do elemento 24.

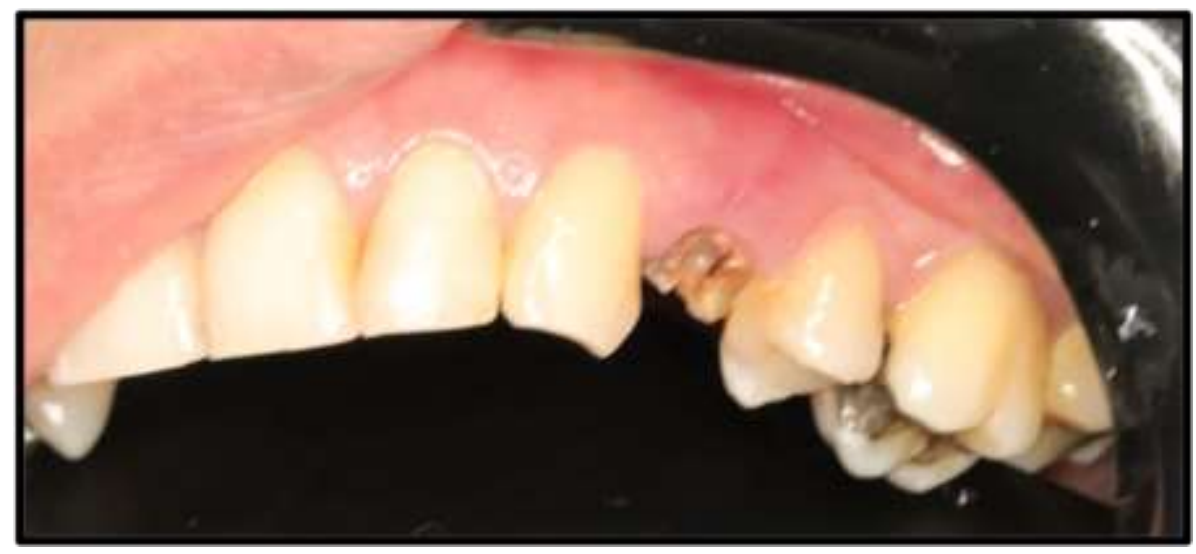

Fonte: Autores.

Foram solicitados exames de sangue e uma tomografia computadorizada (TC), em que foi constatado, a fratura dentária e uma pequena lesão periapical. Também foi observado que havia uma quantidade óssea na região apical que possibilitaria a instalação de um implante após a exodontia do 24 (Figura 2).

Figura 2: Tomografia Computadorizada de feixe cônico com os cortes da região do elemento 24, podendo ser observada a fratura dentária, lesão periapical e remanescente ósseo periapical.

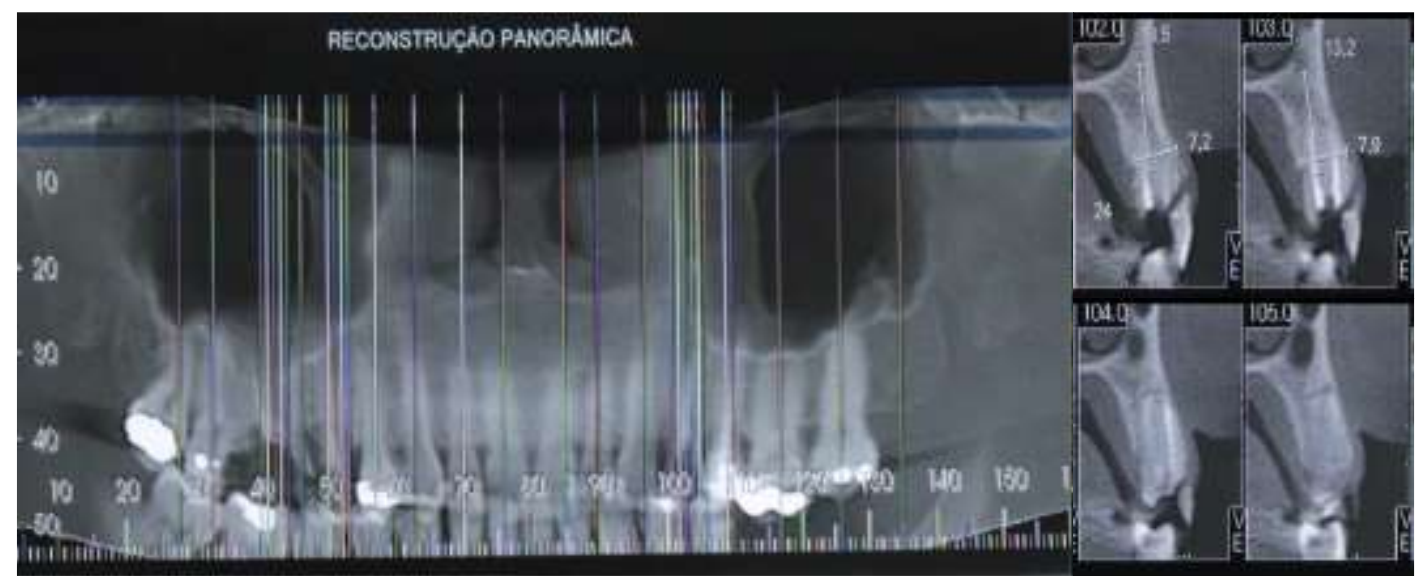

Fonte: Autores.

Visando o restabelecimento da saúde bucal e função mastigatória, foi proposto a paciente a remoção do resto radicular do 24, para instalação de 1 implante e caso fosse atingida o torque necessário, a colocação de uma coroa em função imediata seria realizada.

Após a paciente aceitar o tratamento proposto, o trabalho foi submetido a comissão de ética da Universidade Santo Amaro e aprovado sob o número: 5.046.993. A paciente assinou o termo de consentimento livre e esclarecido (TCLE) e todos os princípios éticos foram respeitados, seguindo a Declaração de Hellsing.

Inicialmente foi realizada uma moldagem com alginato, superior e inferior, para confecção de modelos de estudo, como também foi registrada a relação de mordida. Com a confecção dos modelos, o planejamento foi finalizado, assim como a confecção do guia cirúrgico, o qual utilizou dente de estoque, com a cor A2 (escala Vita Classical: A1-D4), já que este guia servirá futuramente como coroa, a ser cimentada sobre o intermediário. 
Após a anestesia, utilizando a técnica infiltrativa com o uso de anestésico local (Mepivalem ${ }^{\circledR}$ AD 2\%), na região vestibular e palatina, foi realizada uma incisão sulcular com uma lâmina de bisturi número 15 , na vestibular e na palatina do dente 24 , sem a necessidade de realizar incisões relaxantes e nem o descolamento mucoperiostal, desta maneira provocando menos trauma aos tecidos circundantes. Com um cinzel periótomo flexível reto (Millennium-Golgran ${ }^{\circledR}$ ), foram rompidos os ligamentos periodontais (Figura 3a). O dente 24 foi removido com a utilização de um fórceps número 101 (MillenniumGolgran ${ }^{\circledR}$ ) (Figura 3b).

Figura 3: 3a: Rompimento dos ligamentos periodontais com periótomo; 3b: Remoção do elemento dentário 24 com fórceps.

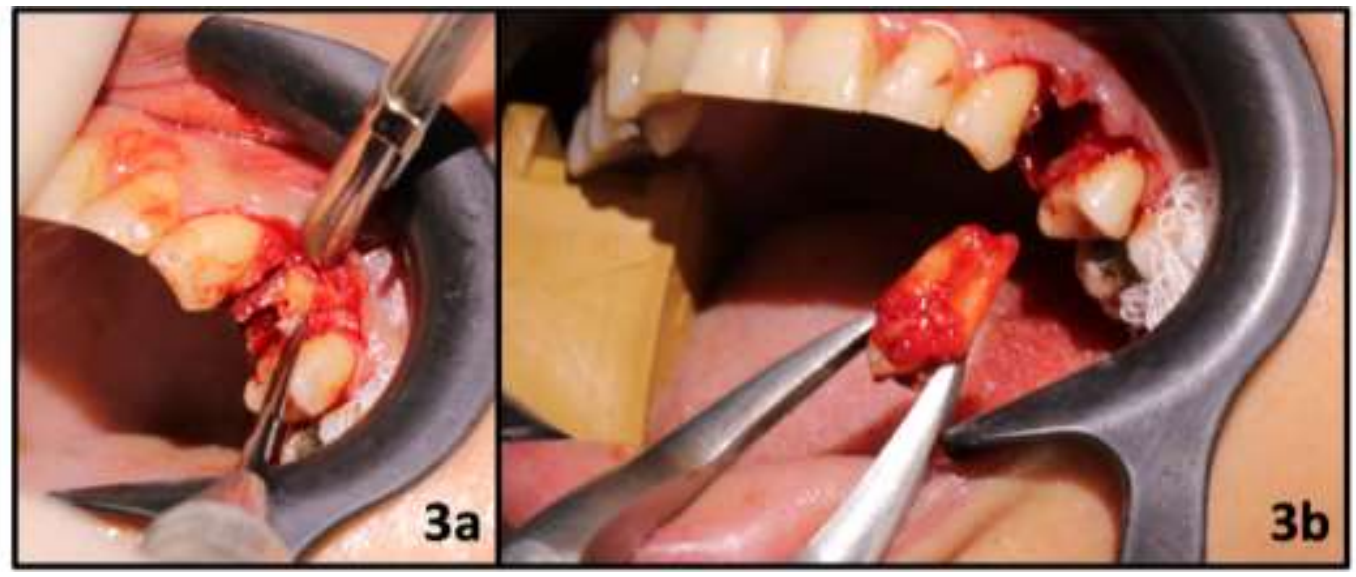

Fonte: O Autor.

Após curetagem da região e abundante irrigação com soro fisiológico, foi utilizado o guia cirúrgico, para a realização da fresagem. As perfurações para instalação do implante, foram realizadas com velocidade $900 \mathrm{rpm}$ e abundante irrigação com soro fisiológico. O sistema utilizado para realizar as perfurações foi o de osteo expansão (Maximus Bone Expander ${ }^{\circledR}$ ), seguindo a sequência determinada pelo fabricante (Figura 4a). Em seguida, foi inserido o implante Cone Morse de 4.3x13,0 mm (Neodent Drive CM Acqua ${ }^{\circledR}$ ) obtendo um torque de 45 N.cm, posicionado $2 \mathrm{~mm}$ infra-ósseo, seguindo a indicação do fabricante (Figura $4 \mathrm{~b})$.

Figura 4: 4a: Guia cirúrgico em posição para realização da fresagem; 4b: Implante em posição, podendo ser observado o gap na vestibular.

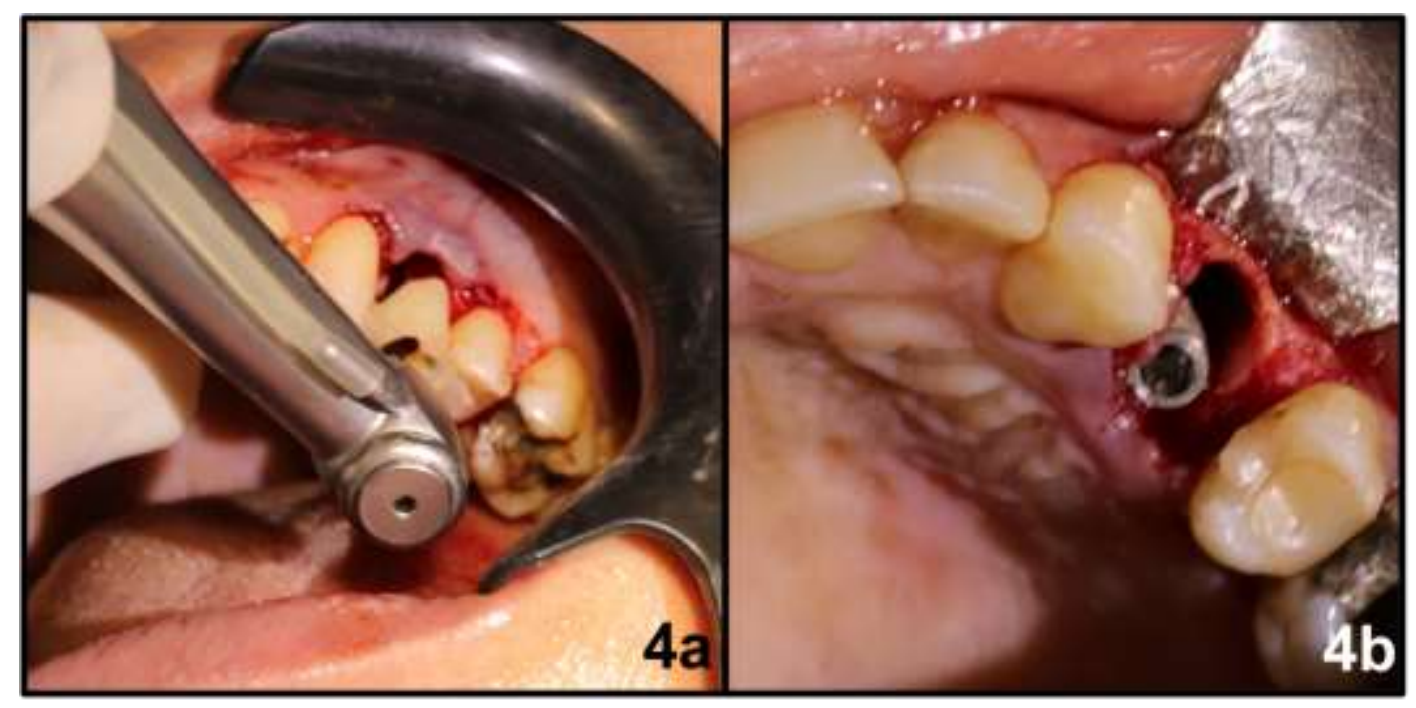

Fonte: Autores. 
O gap formado após instalação do implante, na região vestibular, foi preenchido com um biomaterial xenógeno (Lumina-Bone Médio Critéria ${ }^{\circledR}$ ), juntamente com uma membrana de colágeno (Lumina-coat Criyteria ${ }^{\circledR}$ ) (Figura 5a). Como foi

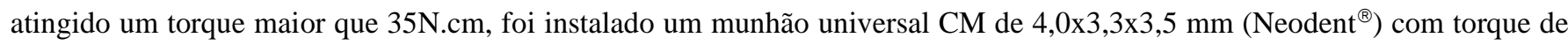
32 N.cm. Em seguida foi colocado um cilindro provisório do munhão universal $\left(\right.$ Neodent $\left.^{\circledR}\right)$ para realizar a sutura, utilizado fio 5.0 mononylon (Best Care ${ }^{\circledR}$ ) (Figura 5b).

Figura 5: 5a: Enxerto posicionado na região do Gap; 5b: Cilindro de proteção posicionado sobre intermediário para realização da sutura.

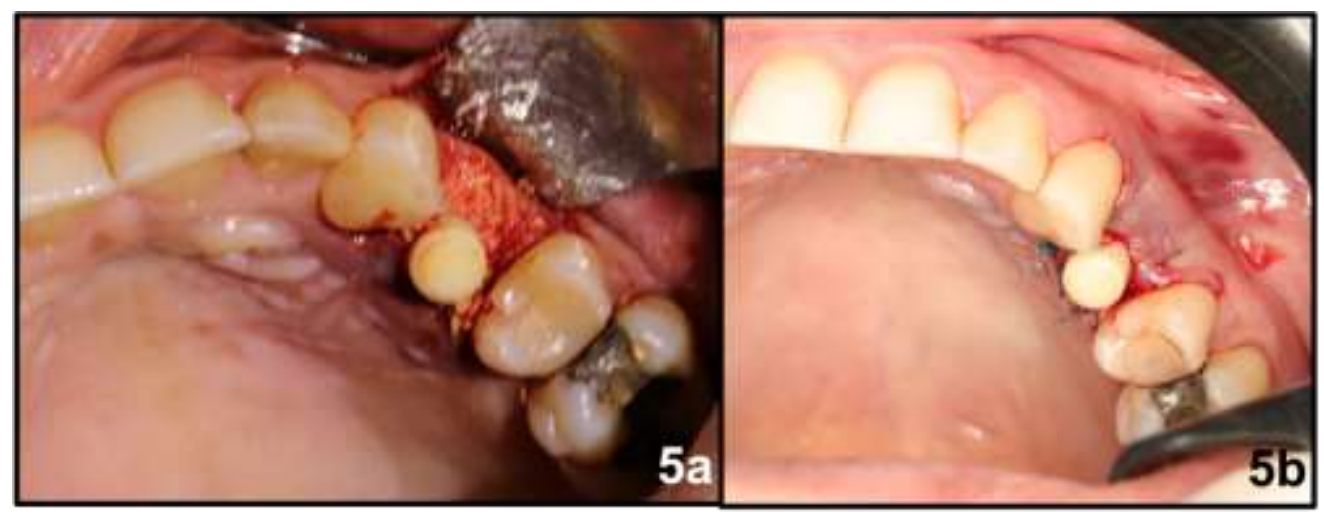

Fonte: Autores.

Ao finalizar a fase cirúrgica, foi utilizada a coroa em resina do guia cirúrgico, sendo cimentada sobre o cilindro provisório do munhão universal com cimento resinoso dual, já em sua posição ideal. Em seguida sendo cimentado com cimento provisório (Provy Dentsply ${ }^{\circledR}$ ) sobre o munhão. Reestabelecendo desta maneira a função imediatamente após a cirurgia, que havia sido perdida devido a fratura do 24 (Figura 6a e 6b).

Figura 6: 6a: Antes do procedimento; 6b: Após o procedimento.

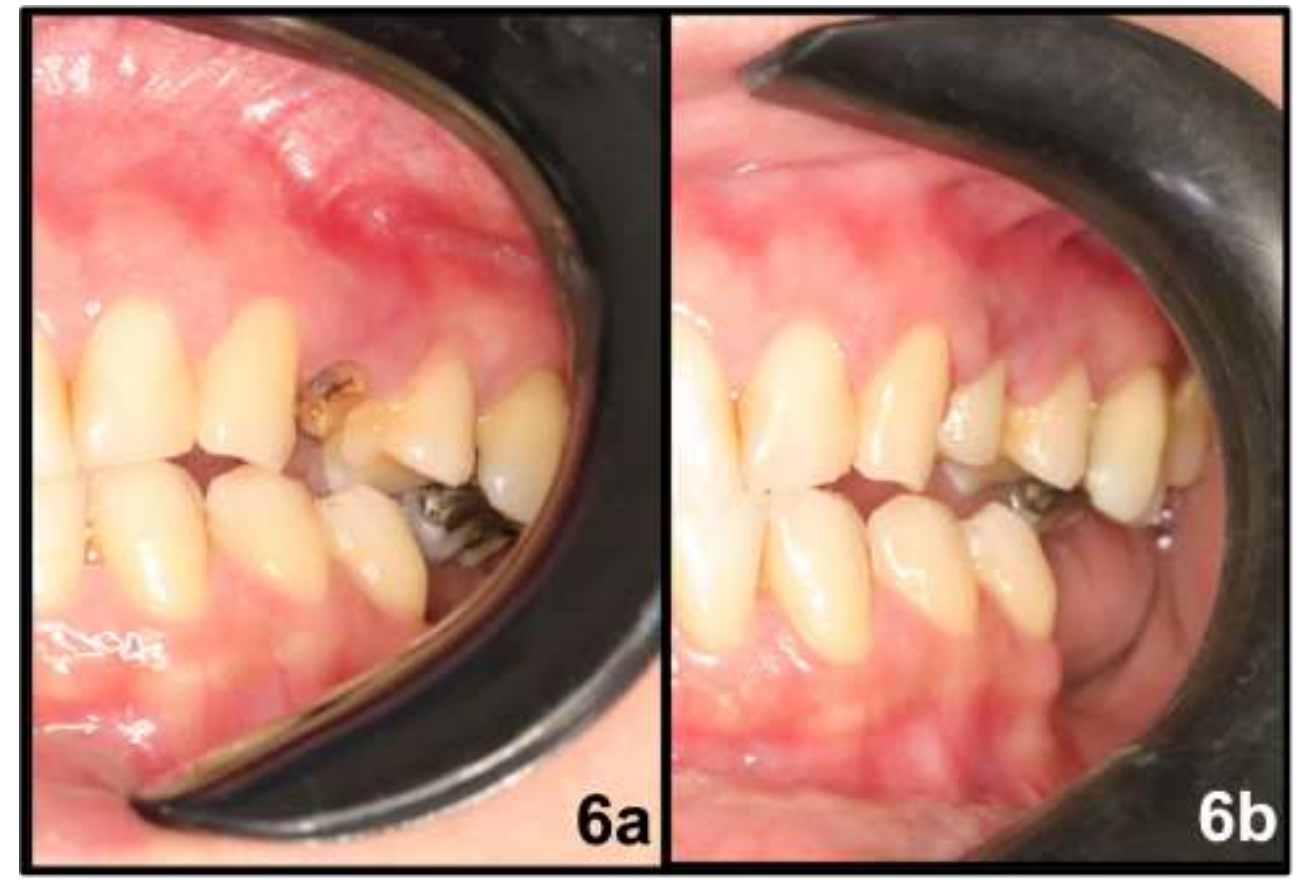

Fonte: O Autor. 
Após conclusão do procedimento, foi receitado antibiótico (Velamox BD 875 ${ }^{\circledR} 12 / 12$ horas por 7 dias), antiinflamatório (Nimesulid $^{\circledR}$ 100mg de 12/12 horas por 5 dias) e uma solução para lavagem bucal (Periogard ${ }^{\circledR}$ 12/12 horas por 7 dias).

\section{Discussão}

A paciente apresentava necessidade de remoção do elemento 24, devido a uma fratura, necessitando desta forma de uma reposição deste dente. Segundo Medeiros, et al., (2020), os pacientes que sofrem a perda de um elemento dentário, devem ser reabilitados de forma imediata, para que o paciente não venha a ser afetado no seu convívio social, devido a estética, como também funcionalmente, pela deficiência da mastigação. Os profissionais de saúde devem estar atentos, já que alterações físicas podem ocasionar traumas psicológicos, podendo interferir diretamente na vida do paciente (Morello, et al., 2011).

$\mathrm{Na}$ avaliação radiográfica da tomografia foi observado, além da fratura dentária, uma lesão periapical. Em um estudo realizado por Mattos, et al., (2018), foram realizadas exodontias seguidas de instalação de implantes imediatos, em dentes com lesões periapicais, sendo constatado que 97,4\% de sucesso, não modificando o período de osseointegração e nem a qualidade da mesma. Seguindo esta mesma linha de raciocínio, Neto, (2017), relata que o índice de sucesso em sítios infectado ou não, se assemelham.

$\mathrm{Na}$ exodontia não foi realizado retalho cirúrgico para preservação dos tecidos adjacentes. A ausência de retalhos favorece a estética, como também a reparação dos tecidos e conforto do paciente (Villaça, et al., 2015). Uma exodontia atraumática deve ser realizada com a intenção de resguardar os tecidos ósseos (Zani, et al., 2011). Deve-se ressaltar que cirurgias quando são minimamente invasivas, ou seja, com ausência de descolamentos e incisões, proporcionam uma menor perda óssea. (Andreiuolo, et al., 2016)

Após a remoção do dente, foi instalado um implante com função imediata. O protocolo inicial de Branemark relata que após exodontia de um elemento condenado, é recomendado esperar um período de 9 a 12 meses para a realização da instalação do implante, porém atualmente sabe-se que se aguardar este período, ocorrerá uma reabsorção alveolar que influenciará tanto nos tecidos moles quanto nos duros, o que levará a uma futura dificuldade na hora de instalar os implantes nesta região (Villaça, et al., 2015). Implantes imediatos são implantes instalados logo após a exodontia de um elemento dentário, devendo ser avaliado individualmente quanto as suas indicações e contra-indicações (Neto, 2017). Implantes dentários instalados no mesmo momento cirúrgico da exodontia, resultam em um menor número de intervenções cirúrgicas e um maior benefício com relação a osseointegração (Geremias, et al., 2015; Medeiros, et al., 2020). São indicações para implantes imediatos: dentes fraturados, com falhas irreversíveis no tratamento endodôntico e com doença periodontal avançada (Zani, et al., 2011). São contra-indicações dos implantes imediatos: higiene bucal deficiente, alcoólatras, fumantes e usuários de drogas ilícitas, assim como pacientes submetidos a tratamentos oncológicos ou que tenham alguma comorbidade sistêmica (Martins, et al., 2020). Um dos avanços que possibilitou o uso da técnica de implante imediato, além da melhoria das técnicas foi os tratamentos de superfície dos implantes (Zani, et al., 2011). Segundo Sampaio, et al., (2020), para se obter o sucesso em implantes imediatos alguns princípios devem ser observados tais como: alvéolo cirúrgico integro, uma adequada cortica óssea vestibular.

O implante instalado obteve um torque inicial de $45 \mathrm{~N} . \mathrm{cm}$, desta maneira foi instalado uma coroa em resina na região. O momento de instalar a prótese esta diretamente relacionada a estabilidade primária, se os implante apresentaram uma boa estabilidade as coroas podem ser instaladas (Zani, et al., 2011). Os implantes imediatos estavam inicialmente relacionados a osseointegração e estabilidade primária. Um fator essencial, para obter sucesso na carga imediata, é o torque de no mínimo 35N.cm (Juodzbalys \& Wang, 2010). Vasconcelos, et al., (2004), relata que é imprescindível a obtenção da estabilidade primária para o uso da função imediata, devendo esta atingir entre 30 e $40 \mathrm{~N} . \mathrm{cm}$, a não obtenção da estabilidade primária 
poderá levar a micromovimentações que darão origem a formação de tecido fibrose na interface implante/osso que acarretará na perda deste implante. Uma ancoragem reduzida e deficiência do processo de osseointegração pode ocorrer quando se opta por implante imediato, este fato pode vir a ocorrer devido a existência de um GAP, o qual pode interferir na estabilidade inicial ou até no resultado protético final (Miranda e Neto, 2019). A previsibilidade da instalação imediata de próteses sobre implantes ainda possui controvérsias, porém através do planejamento reverso, o componente protético visa além da estética pósoperatória, benefícios com relação a preservação dos tecidos moles (Sampaio, et al., 2020).

Logo em seguida da instalação do implante, foi observado um GAP na porção vestibular, sendo preenchido com enxerto ósseo xenógeno, finalizando com o uso de uma membrana. A utilização de implantes imediatos associados a enxerto ósseo deve ser feito sempre que houver a indicação, já que este dispensará procedimentos regenerativos futuros (Andreiuolo, et al., 2016). Um resultado positivo, com o uso da técnica dos implantes imediatos, está diretamente relacionado com a correta posição tridimensional do implante, desta forma nunca posicionando os implantes no centro do alvéolo (Mattos, et al., 2018). Quando há presença de GAP's, deve ser feito uso biometariais, com o objetivo de uma neoformação óssea para assim prevenir um colapso do tecido ósseo e consequentemente do tecido peri-implantar (Vasconcelos, et al., 2016; Mattos, et al., 2018).

\section{Conclusão}

A função imediata com o uso de implantes dentários após exodontia é possível, como foi demonstrado no caso clínico. É uma técnica favorável que proporcionou o sucesso na reabilitação, devolvendo a função e estética imediata ao paciente. devendo o cirurgião-dentista realizar sempre o planejamento reverso, estando atento as indicações e contraindicações da técnica. Estudos clínicos bem desenhados ainda são necessários para comparar diretamente o efeito do momento da colocação do implante na saúde peri-implantar e na estabilidade dos tecidos duros e moles peri-implantares, sugerindo novos trabalhos abordando estes tópicos.

\section{Referências}

Adell, R., Lekhom, U., Rockler, B., \& Branemark, PI. (1981). A 15-years study of osseointegrated implants in the treatment of the edentulous jaw. Int J Oral Surg, 10(6), 387-416.

Andersen, E., Haanaes, H. R., \& Knutsen, B. M. (2002) Immediate loading of single-tooth ITI implants in the anterior maxilla: a prospective 5-year pilot study. Clin Oral Impl, 13(3), 281-7.

Andreiuolo, R., Vasconcellos, F., Andrade, A., Groisman, M., \& Júnior, G. (2016). Implante imediato na região anterior: aspectos cirúrgicos e protéticos. Rev. bras. odontol., 73 (1), 84-88. http://revista.aborj.org.br/index.php/rbo/article/view/682/492

Bahat, O. (2000). Branemark system implants in the posterior maxilla: clinical study of 660 implants follow for 5 to 12 years. Int J Oral Maxillofacial Implants; 15(15), 646-53.

Bassir, S. H., El Kholy, K., Chen, C. Y., Lee, K. H., \& Intini, G. (2018). Outcome of early dental implant placement versus other dental implant placement protocols: A systematic review and meta-analysis. J Periodontol, May, 90(5), 493-506. 10.1002/JPER.18-0338. Epub 2018 Dec 5. PMID: 30395355; PMCID: PMC6500770.

Becker, W. (2005). Immediate implant placement: diagnosis, treatment planning and treatment steps/or successful outcomes. J Calif Dent Assoc, 33(4), 30310 .

Branemark, P. I., Adell, R., Breine, U., Hansonn, B. O., Lindstron, J., \& Ohloson, A. (1969). Intra- osseous anchorage of dental prostheses I. Experimental studies. Scand J Plast Reconstr Surg, 3(2), 81-100.

Brunski, J. B. (1992). Biomechanical factors affecting the bone-dental implant interface. Clin Mater, 10(3), $153-201$.

Ericsson, I., Nilson, H., Lindh, T., Nilner, K., \& Randow, K. (2000). Immediate functional loading of Branemark dental implants. An 18-month clinical follow-up study. Clin Oral Implants Res, 11(1), 26-33.

Geremias, T., Montero, J., Juanito, G., Morsch C., Rafael, C., \& Magini, R. (2015). Regeneração da parede vestibular em implante anterior com uso de BioOss ${ }^{\circledR}$ - relato de caso. Full Dent. Sci. Santa Catarina, 6 (24), 486-491. https://www.researchgate.net/profile/Gabriella-Mercedes-PenarrietaJuanito/publication/304668463_Buccal_wall_regeneration_in_an_aesthetic_implant_region_using_Bio-OssR/links/58aee73892851cf7ae88ef57/Buccal-wallregeneration-in-an-aesthetic-implant-region-using-Bio-OssR.pdf 
Haas, R., Mendsdorff-Poully, N., Mailath, G., \& Watsek, G. (1995). Brane- mark single tooth implants: a preliminary report of 76 im- plants. J Prosthet Dent, 73(3), 274-9.

Juodzbalys, G., \& Wang, H-L. (2010). Socket Morphology--Based Treatment for Implant Esthetics: A Pilot Study. Int J Oral Maxillofac Implants, 25(5), 970978. [PubMed: 20862411]

Leary, J. C., \& Hirayama, M. (2003). Extraction, immediate-load implants, impressions and final restorations in two patient visits. J Am Dent Assoc, 134(6), $715-20$.

Martins, I., Pedraça, V., \& Filho, M., (2020). Reabilitação oral com implante imediato: revisão de literatura. Brazilian Journal of Development, Curitiba, 6 (12), 95785-95794. https://www.brazilianjournals.com/index.php/BRJD/article/view/21215/16916

Mattos, T., Mendes, F., Suzuki, T., Gulinelli, J., Galito, P., \& Santos, P. (2018). Implante imediato associado à infecção periapical crônica: relato de caso clinico. Arch Health Invest, Minas Gerais, 7 (5), 200-204. https://archhealthinvestigation.com.br/ArcHI/article/view/2994/pdf

Medeiros, M., Marcelino, K., Júnior, J., Pinheiro, N., Freire, J., Dantas, E. M., Barbosa, G. A. S., Gondim, A. L. M. F., Ribeiro, E. D., \& Almeida Neto, L. F. (2020). Exodontia atraumática e implante imediato em área estética: relato de caso. Research, Society and Development, 9 (9), 01-15. https://rsdjournal.org/index.php/rsd/article/view/7997/7153

Miranda, R. C., \& Neto, M. A. F. (2019). Plasma rico em fibrina para implante imediato: Revisão de Literatura. Id on Line Rev. Mult. Psic., Bahia, 13 (47), 889-899. https://idonline.emnuvens.com.br/id/article/view/2092/3175

Morello, J., Ribeiro, F., Roldi, A., Pereira, R., Barroso, J., \& Intra, J. (2011). Sequelas subsequentes aos traumatismos dentários com envolvimento endodôntico. Revista Brasileira de Pesquisa em Saúde, 13 (2), 68-73. file:///C:/Users/Paula/Downloads/1605-Texto\%20do\%20artigo-2510-1-1020110906\%20(12).pdf

Neto, F. J. S. (2017). Instalação de implante imediato em sítio infectado: revisão de literatura. Monografia (Bacharel em Odontologia) - Faculdade Maria Milza, Governador Mangabeira, Bahia.

http://131.0.244.66:8082/jspui/bitstream/123456789/481/1/Monografia\%20Fernando\%20Jos\%c3\%a9\%20_Odontologia\%202017_.pdf

Sampaio, V., Silva, D., Barreiro, F., Brito, H., Andrade, F., \& Gomes, D. (2020). Implante imediato associado a enxerto xenógeno e provisionalização imediata em área infectada: relato de caso. Arch Health Invest, Paraíba, 9 (5), 444-448. https://archhealthinvestigation.com.br/ArcHI/article/view/4784/pdf

Piattelli, A., Ruggeri, A., Franchi, M., Romasco, N., \& Trisi, P. (1993). An his- tologic and histomorphometric study of bone reactions to un- loaded and loaded non-submerged single implants in monkeys: a pilot study. J Oral Implantol, 19(4), 314-20.

Popper, H.A., Popper, M. J., \& Popper, J. P. (2003). The branemark novum protocol: description of the treatment procedure and a clinical pilot study of 11 cases. Int J Periodontics Restorative Dent, 23(5), 459-65.

Schropp, L., Wenzel, A., Kostopoulos, L., \& Karring, T. (2003). Bone healing and soft tissue contour changes following single-tooth extrac- tion: a clinical and radiographic 12-month prospective study. Int J Periodontics Restorative Dent, 23(4), 313-23.

Vasconcelos, L., Hiramatsu, D., Paleckis, L., Francischone, C., Vasconcelos, R., \& Chaves, T. (2016). Implante imediato e preservação de alvéolo com BioOss Collagen® em área estética. The International Journal of Oral \& Maxillofacial. São Paulo, 1(3), 01-09.

https://www.researchgate.net/profile/Laura-Paleckis/publication/304813721_Implante_Imediato_e_Preservacao_de_Alveolo_com_Bio-Oss_CollagenR_em_ Area_Estetica/links/5b3038450f7e9b0df5c6e0d1/Implante-Imediato-e-Preservacao-de-Alveolo-com-Bio-Oss-CollagenR-em-Area-Estetica.pdf

Villaça, J. H., Pesqueira, E. I. De O., \& Guimarães, C. M. (2015). Relato de caso clínico de implante e provisionalização imediatos com um inovador sistema de cirurgia guiada - benefícios e avaliação da acurácia. Prótese News, 2 (2), 180-191. https://pesquisa.bvsalud.org/portal/resource/pt/biblio-853949?lang=fr

Zani, S, Alves, R., Korb, S., Rivaldo, E., \& Frasca, L. (2011). Colocação de implante imediato após exodontia: relato de caso clínico. Odontologia ClínicoCientífica, 10 (3), 281-284. http://revodonto.bvsalud.org/pdf/occ/v10n3/a18v10n3.pdf 